\title{
TIME-VARYING EXTREME VALUE DEPENDENCE WITH APPLICATION TO LEADING EUROPEAN STOCK MARKETS ${ }^{1}$
}

\author{
By Daniela Castro-Camilo, Miguel de Carvalho and \\ JENNIFER WADSWORTH \\ King Abdullah University of Science and Technology, \\ University of Edinburgh, and Lancaster University
}

\begin{abstract}
Extremal dependence between international stock markets is of particular interest in today's global financial landscape. However, previous studies have shown this dependence is not necessarily stationary over time. We concern ourselves with modeling extreme value dependence when that dependence is changing over time, or other suitable covariate. Working within a framework of asymptotic dependence, we introduce a regression model for the angular density of a bivariate extreme value distribution that allows us to assess how extremal dependence evolves over a covariate. We apply the proposed model to assess the dynamics governing extremal dependence of some leading European stock markets over the last three decades, and find evidence of an increase in extremal dependence over recent years.
\end{abstract}

1. Introduction. In recent years, international stock markets have been registering unprecedented levels of turbulence. Episodes such as the subprime crisis and the Greek debt crisis may have boosted this turbulence a little further, and led many to fear a financial doomsday. The situation has been extraordinarily delicate in Europe, where evidence of increasing extremal dependence was found by Poon, Rockinger and Tawn $(2003,2004)$ before the most recent financial crisis. We look to update suitable parts of their analysis and in particular analyze the time-varying extremal dependence in a more complete manner than has been done before. To achieve this goal, we propose an approach for modeling nonstationarity in the extreme value dependence structure.

Statistical modeling of univariate extreme values has been in development since the 1970s [Natural Environment Research Council (1975)]. Fundamental to practical application to complex problems has been the development of methodology to account for nonstationarity in the distributions of interest, which was first strongly advocated by Davison and Smith (1990). Typical approaches to this problem are based around the generalized linear modeling idea of allowing the parameters of a

Received March 2017; revised June 2017.

${ }^{1}$ Supported in part by Fundação para a Ciência e a Tecnologia, through UID/MAT/00006/2013 and by the Chilean National Science Foundation through Fondecyt 11121186, "Constrained Inference Problems in Extreme Value Modeling".

Key words and phrases. Angular measure, bivariate extreme values, European stock market integration, risk, statistics of extremes. 
marginal distribution to depend on covariates; more flexible approaches involving generalized additive modeling were introduced by Chavez-Demoulin and Davison (2005). Eastoe and Tawn (2009) present related ideas where data are preprocessed according to their dependence on covariates.

Statistical methods for modeling multivariate extreme values were introduced by Tawn (1988), and developed in Tawn (1990) and Coles and Tawn (1991). Since this time, much work has been done on developing dependence modeling frameworks for extremes, yet surprisingly little has focused on how to incorporate nonstationarity into the (extremal) dependence structure. Exceptions include Eastoe (2009), who introduces a conditionally independent hierarchical model, Jonathan, Ewans and Randell (2014), who develop methodology for including covariates in the model of Heffernan and Tawn (2004), and de Carvalho and Davison (2014), who develop a semiparametric model for settings where several multivariate extremal distributions are linked through the action of a covariate on an unspecified baseline distribution. In addition, Huser and Genton (2016) developed nonstationary models for spatial extremes where covariates can be included. In this work, we add to the literature on modeling nonstationarity in the dependence structure by proposing flexible methodology for a simple set-up. Working within a tail dependence framework known as asymptotic dependence, we suppose that the relevant bivariate extreme value distribution evolves over a certain covariate of interest. The approach that we take is fully nonparametric, which is advantageous since neither the form of the bivariate distribution at a given covariate, nor the form of dependence on the covariate can be parametrically specified.

Our methodology is particularly tailored for assessing temporal changes in extremal dependence, which is the situation that we would like to investigate in our motivating example. Poon, Rockinger and Tawn $(2003,2004)$ studied the dependence between stock market returns in the US, UK, France, Germany, and Japan. The main focus of their works was to highlight that not all markets exhibit a sufficient strength of tail dependence to be asymptotically dependent, and to propose alternative dependence summaries. However, considering only the European markets, they noted that there was evidence for relatively strong left-tail dependence, and we also find evidence for asymptotic dependence in the left tails of these major European markets. As noted by Poon, Rockinger and Tawn (2003), the dependence is not stationary in time, and a main focus of this work is to explore this nonstationarity using a full model for the time-varying dependence structure, rather than simply summary statistics.

In the next section, we provide a background on dependence modeling for extreme values, and introduce our proposed framework for incorporating nonstationarity. In Section 3, we introduce our estimation and inference methods; numerical illustrations follow in Section 4. The focus of Section 5 is on applying the proposed methods to returns from three major European stock markets-using CAC, DAX, and FTSE - to assess the evolution of their extremal dependence structure over time. We conclude in Section 6. 


\section{Conditional modeling for bivariate extremes.}

2.1. Bivariate statistics of extremes. Let $\left\{\left(Y_{i, 1}, Y_{i, 2}\right)\right\}_{i=1}^{N}$ be a collection of independent and identically distributed random vectors with continuous marginal distributions $F_{Y_{1}}$ and $F_{Y_{2}}$. We are concerned with assessments of the extremal dependence between the components of the vectors, and thus without loss of generality we shall suppose that they have standard Fréchet margins, that is, $\mathrm{P}\left(Y_{j}>y\right)=\exp (-1 / y)$, for $y>0$ and $j=1,2$. Let

$$
\left(M_{N, 1}, M_{N, 2}\right)=\frac{1}{N}\left(\max _{1 \leq i \leq N}\left\{Y_{i, 1}\right\}, \max _{1 \leq i \leq N}\left\{Y_{i, 2}\right\}\right)
$$

be the standardized vector of componentwise maxima. Then if

$$
\mathrm{P}\left(M_{N, 1} \leq y_{1}, M_{N, 2} \leq y_{2}\right) \rightarrow G\left(y_{1}, y_{2}\right), \quad \text { as } N \rightarrow \infty,
$$

where $G$ is a nondegenerate distribution function, $G$ has the form:

$$
G\left(y_{1}, y_{2}\right)=\exp \left\{-2 \int_{[0,1]} \max \left(\frac{w}{y_{1}}, \frac{1-w}{y_{2}}\right) H(\mathrm{~d} w)\right\}, \quad y_{1}, y_{2}>0 .
$$

Here, $G\left(y_{1}, y_{2}\right)$ is the so-called bivariate extreme value distribution and $H$ is a probability measure-known as the angular measure. A consequence of Pickands' (1981) representation theorem is that the angular measure needs to obey the following marginal moment constraint

$$
\int_{[0,1]} w H(\mathrm{~d} w)=1 / 2
$$

see, for example, Coles (2001), Theorem 8.1. Let $R=Y_{1}+Y_{2}$ and $W=Y_{1} /\left(Y_{1}+\right.$ $Y_{2}$ ). de Haan and Resnick (1977) have shown that the convergence in (2.1) is equivalent to

$$
\mathrm{P}(W \in \cdot \mid R>u) \rightarrow H(\cdot), \quad u \rightarrow \infty .
$$

In practice, convergence (2.4) is more often useful than (2.1) and tells us that when the "radius" $R$ is large, the "pseudo-angles" $W$ are approximately distributed according to $H$, and approximately independent of $R$. The distribution of mass of $H$ on $[0,1]$ describes the extremal dependence structure of the random vector $\left(Y_{1}, Y_{2}\right)$. The extreme cases of this distribution are given by asymptotic independence, whereby all mass is placed at the vertices of $[0,1]$, giving $G\left(y_{1}, y_{2}\right)=\exp \left\{-\left(y_{1}^{-1}+y_{2}^{-1}\right)\right\}$, and by complete dependence, whereby all mass is placed at the center of the interval, yielding $G\left(y_{1}, y_{2}\right)=\exp \left\{-\max \left(y_{1}^{-1}, y_{2}^{-1}\right)\right\}$. We refer to situations where $H$ has mass away from the vertices as asymptotic dependence and this will be the framework of our modeling. Nevertheless, asymptotic independence is a relatively common situation in practice, and can be detected when $R$ and $W$ are not found to be independent for any values of $R$, with the mass of $W$ moving closer to 0 and 1 as events become more extreme. In this situation, no 
models for $H$ will provide useful information on the extremal dependence structure. Finally, a standard assumption for statistical modeling is that $H$ is absolutely continuous with angular density $h=\mathrm{d} H / \mathrm{d} w$, and this will be our framework.

Functionals of interest of the angular measure include the bivariate extreme value distribution (2.2), which also represents the extreme value copula, $C_{\mathrm{EV}}$, [e.g., Gudendorf and Segers (2010)] in Fréchet margins, that is, $G\left(y_{1}, y_{2}\right)=$ $C_{\mathrm{EV}}\left(\mathrm{e}^{-1 / y_{1}}, \mathrm{e}^{-1 / y_{2}}\right)$. Other functionals include the Pickands (1981) dependence function $A(w)=1-w+2 \int_{0}^{w} H(u) \mathrm{d} u$, and the extremal coefficient $C=$ $2 A(1 / 2)$. Extreme value independence corresponds to $A(w)=1$, whereas perfect dependence corresponds to $A(w)=\max (w, 1-w)$.

2.2. Conditional modeling framework. We define the conditional bivariate extreme value (BEV) distribution as

$$
\begin{aligned}
G_{x}\left(y_{1}, y_{2}\right) & \equiv G\left(y_{1}, y_{2} \mid X=x\right) \\
& =\exp \left\{-2 \int_{[0,1]} \max \left(\frac{w}{y_{1}}, \frac{1-w}{y_{2}}\right) H(\mathrm{~d} w \mid X=x)\right\},
\end{aligned}
$$

for $x \in \mathcal{X} \subseteq \mathbb{R}$, and $y_{1}, y_{2}>0$. Here, $H_{x}(\cdot) \equiv H(\cdot \mid X=x)$ are conditional probability measures satisfying

$$
\int_{[0,1]} w H_{x}(\mathrm{~d} w)=1 / 2, \quad x \in \mathcal{X} .
$$

If $H_{x}(w) \equiv H_{x}[0, w]$ is absolutely continuous, its conditional angular density is $h_{x}=\mathrm{d} H_{x} / \mathrm{d} w$. Further aspects of conditional angular measures are discussed in de Carvalho (2017).

Our main modeling object of interest will be the set of conditional angular densities $\left\{h_{x}(w): w \in[0,1], x \in \mathcal{X}\right\}$, which we will refer to as the angular surface. A simple angular surface can be obtained with the conditional angular density $h_{x}(w)=\beta\left(w ; \mu_{x}, \mu_{x}\right)$, where $\mu: \mathcal{X} \mapsto(0, \infty)$, and $\beta(\cdot ; p, q)$ denotes the beta density with shape parameters $p, q>0$. In Figure 1(a), we represent an angular surface based on this model, with $\mu_{x}=x$, for $x \in \mathcal{X}=[0.5,50]$. As can be seen, larger values of the predictor $x$ lead to stronger levels of extremal dependence. Other angular surfaces can be readily constructed from parametric models for the angular density.

EXAMPLE 1 (Conditional logistic model). The logistic angular surface is a covariate-adjusted extension of the logistic model [Coles (2001), page 146], and it is based on the conditional angular density

$$
\begin{aligned}
h_{x}(w)= & \frac{1}{2}\left(\frac{1}{\alpha_{x}}-1\right)\{w(1-w)\}^{-1-1 / \alpha_{x}} \\
& \times\left\{w^{-1 / \alpha_{x}}+(1-w)^{-1 / \alpha_{x}}\right\}^{\alpha_{x}-2},
\end{aligned}
$$



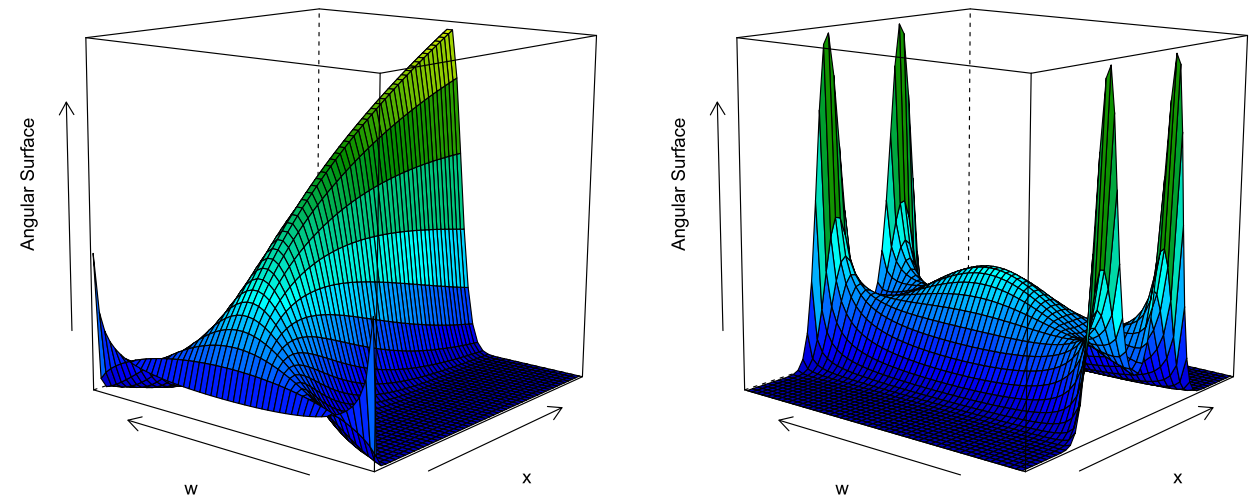

FIG. 1. (a) Angular surface from a conditional beta family, with $\mu_{x}=x$, for $x \in \mathcal{X}=[0.5,50]$. (b) Angular surface from a conditional logistic family, with $\alpha_{x}=\Phi\left(x^{2}\right)$, for $x \in \mathcal{X}=[-3,3]$.

for $w \in(0,1)$, where $\alpha: \mathcal{X} \mapsto(0,1]$. The closer $\alpha_{x}$ is to 0 , the higher the level of extremal dependence, while the closer $\alpha_{x}$ is to 1 , the closer we get to independence. Angular surfaces with simple "shapes" can be obtained by modeling $\alpha_{x}$ with either a distribution function, $F(x)$, or a survivor function, $1-F(x)$. More sophisticated shapes can be obtained with $\alpha_{x}=(F \circ G)(x)$, for a certain continuous function $G$ : $\mathcal{X} \mapsto \mathbb{R}$. In Figure 1(b), we represent the logistic angular surface in (2.7) with $\alpha_{x}=$ $\Phi\left(x^{2}\right)$, for $x \in \mathcal{X}=[-3,3]$, where $\Phi$ denotes the standard normal distribution function.

EXAMPLE 2 (Conditional Dirichlet model). The Dirichlet angular surface is a covariate-adjusted extension of the Dirichlet model [Coles and Tawn (1991)], and it is based on the conditional angular density

$$
h_{x}(w)=\frac{a_{x} b_{x} \Gamma\left(a_{x}+b_{x}+1\right)\left(a_{x} w\right)^{a_{x}-1}\left\{b_{x}(1-w)\right\}^{b_{x}-1}}{2 \Gamma\left(a_{x}\right) \Gamma\left(b_{x}\right)\left\{a_{x} w+b_{x}(1-w)\right\}^{a_{x}+b_{x}+1}}, \quad w \in(0,1),
$$

where $a: \mathcal{X} \mapsto(0, \infty)$ and $b: \mathcal{X} \mapsto(0, \infty)$. Angular surfaces with simple shapes can be obtained with $a_{x}=b_{x}=\exp (x)$, while if more complex dynamics are desirable, can be based on $a_{x}=\exp \{\mathcal{A}(x)\}, b_{x}=\exp \{\mathcal{B}(x)\}$, where $\mathcal{A}: \mathcal{X} \mapsto \mathbb{R}$ and $\mathcal{B}: \mathcal{X} \mapsto \mathbb{R}$ are continuous functions.

The basic idea of a conditional angular measure is not especially complicated, and inference for such would be simple if: (i) we knew our data conform to a particular parametric family, and (ii) we knew precisely how that family depended on $x$. However, since we do not have knowledge of either of these things, the natural approach to take is a nonparametric one. We assume that $h_{x}$ varies smoothly with $x$, and thus kernel smoothing becomes a natural option. We describe our estimation strategy in Section 3. 
2.3. Related conditional objects of interest. Our estimation target $\left\{h_{x}(w)\right.$ : $w \in[0,1], x \in \mathcal{X}\}$ can be used for constructing other objects of interest when modeling bivariate extremes. For example, a conditional version of Pickands (1981) dependence function can be defined as

$$
A_{x}(w)=1-w+2 \int_{0}^{w} H_{x}(u) \mathrm{d} u, \quad x \in \mathcal{X}, w \in[0,1],
$$

leading to the conditional extremal coefficient $C_{x}=2 A_{x}(1 / 2)$. A covariateadjusted extreme value copula can be readily constructed from (2.5). Although much theoretical and applied work has been devoted to time-dependent copulas [Patton (2006), Veraverbeke, Omelka and Gijbels (2011), Acar, Craiu and Yao (2011), Fermanian and Wegkamp (2012)], the amount of work dedicated to timevarying extreme value copulas is by comparison fairly reduced, but of obvious relevance in a wealth of contexts of applied interest. The latter setup is the one of interest in the current manuscript.

EXAMPLE 3. Using the conditional angular density from Example 1, we obtain $A_{x}(w)=\left\{(1-w)^{1 / \alpha_{x}}+w^{1 / \alpha_{x}}\right\}^{\alpha_{x}}$ and $C_{x}=2^{\alpha_{x}}$, while the logistic angular surface is based on the conditional BEV distribution,

$$
G_{x}\left(y_{1}, y_{2}\right)=\exp \left\{-\left(y_{1}^{-1 / \alpha_{x}}+y_{2}^{-1 / \alpha_{x}}\right)^{\alpha_{x}}\right\}, \quad x \in \mathcal{X}, y_{1}, y_{2}>0 .
$$

\section{Estimation and inference.}

3.1. Derivation of pseudo-angles. Consider equation (2.4). We are now supposing nonstationarity in the dependence structure such that

$$
\mathrm{P}(W \in \cdot \mid R>u, X=x) \rightarrow H_{x}(\cdot), \quad u \rightarrow \infty .
$$

Note that we still assume that $R$ and $W$ are derived from $Y_{1}, Y_{2}$ with standard Fréchet margins. Typically, when stationarity in the extremal dependence structure is assumed, one searches for a high threshold in $R$, such that $W$ and $R$ are approximately independent above the threshold, and uses all $W$ associated to threshold exceedances of $R$ for inference. Supposing that $x$ does not impact upon the rate of convergence in the limit (3.1), a similar approach is justified here. However, for prudence, we assess the dependence of $R$ on $x$ using quantile regression [Koenker (2005)]. To be consistent with the nonparametric nature of our approach, we fit a nonparametric quantile regression using regression splines. This method flexibly fits a piecewise cubic polynomial to estimate the $95 \%$ quantile of $R$. If any relationship between $R$ and $x$ is detected, then we take the $W$ associated to exceedances of the fitted threshold by $R$ for inference. Below we use $n=o(N)$ to denote the number of pseudo-angles that resulted from thresholding $R_{i}=Y_{i, 1}+Y_{i, 2}$, for $i=1, \ldots, N$. Further details on the derivation of pseudo-angles for our data application can be found in Section 5.3. 
We note that we are not allowing for the margins to change over the predictor. This is however a sensible modeling assumption for our data application, because (filtered) returns are known to be approximately stationary. Indeed, as posed by Resnick (2007), page 7, "Returns have more attractive statistical properties than prices such as stationarity". See Section 5.2 for details on the filtering methods used in our data application.

3.2. Conditional angular density estimation. Here, we outline our estimator for the family of densities $\left\{h_{x}(w): w \in[0,1], x \in \mathcal{X}\right\}$. Assume observations $\left\{\left(X_{i}, W_{i}\right)\right\}_{i=1}^{n}$, where the covariates $X_{i}$ are continuous and in $\mathcal{X} \subseteq \mathbb{R}$. Let $K_{b}(x)=(1 / b) K(x / b)$ be a kernel with bandwidth $b>0$. For any $x \in \mathcal{X}$, we define the estimator

$$
\widehat{h}_{x}(w)=\sum_{i=1}^{n} \pi_{b, i}(x) \beta\left(w ; v W_{i} \theta_{b}(x)+\tau, v\left\{1-W_{i} \theta_{b}(x)\right\}+\tau\right),
$$

for $w \in(0,1)$, where

$$
\theta_{b}(x)=\frac{1 / 2}{\sum_{i=1}^{n} \pi_{b, i}(x) W_{i}}, \quad \pi_{b, i}(x)=\frac{K_{b}\left(x-X_{i}\right)}{\sum_{j=1}^{n} K_{b}\left(x-X_{j}\right)}, \quad i=1, \ldots, n .
$$

The moment constraint (2.6) is satisfied, since

$$
\int_{0}^{1} w \widehat{h}_{x}(w) \mathrm{d} w=\frac{\sum_{i=1}^{n} K_{b}\left(x-X_{i}\right)\left\{v W_{i} \theta_{b}(x)+\tau\right\}}{(v+2 \tau) \sum_{i=1}^{n} K_{b}\left(x-X_{i}\right)}=\frac{\nu / 2+\tau}{\nu+2 \tau}=1 / 2,
$$

for all valid $\tau \geq 0$, upon substitution of $\theta_{b}(x)$.

The two kernels $\left(K_{b}\right.$ and $\beta$ ) and the three parameters involved in our estimator can be interpreted as follows. The bandwidth $b>0$ is the scale parameter of the kernel $K_{b}$ and controls the amount of smoothing in the $x$-direction. The choice of the kernel $K_{b}$ is subject to the typical considerations. In principle, $K_{b}$ should be symmetric and unimodal, since there is a sense in which density estimators based on kernels that do not satisfy these requirements are inadmissible [Cline (1988)]. While there are many kernel functions that do satisfy these basic requirements, it is well known that the choice of the kernel has little impact on the corresponding estimators; see Wand and Jones (1995), Chapter 2, and references therein. The parameter $v>0$ is asymptotically inversely proportional to the variance of the kernel $\beta$ and has the main role of controlling the amount of smoothing in the $w$-direction. The additional parameter $\tau \geq 0$ has the role of adjusting slightly the center of the kernel, allowing more flexible estimation, whilst not affecting the imposition of the moment constraint. Note that $\tau=0$ yields a kernel with mean equal to $W_{i}$, whilst $\tau=1$ yields a kernel with mode $W_{i}$. In addition, $\theta_{b}(x)$ assesses by how much we deviate from the moment constraint (2.6). To see this, note that $\theta_{b}(x)=(1 / 2) / \widehat{\mathrm{E}}(W \mid X=x)$, where $\widehat{\mathrm{E}}(W \mid X=x)=\sum_{i=1}^{n} \pi_{b, i}(x) W_{i}$ is the Nadaraya-Watson estimator [Nadaraya (1964), Watson (1964)] of $\mathrm{E}(W \mid X=x)=\int_{[0,1]} w H_{x}(\mathrm{~d} w)=1 / 2$, for all $x \in \mathcal{X}$. 
Plug-in estimators for the related conditional objects of interest discussed in Section 2.3 can be readily obtained; particularly

$$
\widehat{H}_{x}(w)=\sum_{i=1}^{n} \pi_{b, i}(x) B\left(w ; v W_{i} \theta_{b}(x)+\tau, v\left\{1-W_{i} \theta_{b}(x)\right\}+\tau\right), \quad w \in(0,1),
$$

where $B(w ; p, q)$ is the regularized incomplete beta function, with $p, q>0$; in addition, the plug-in estimators for the conditional Pickands dependence function, extremal coefficient, and bivariate extreme value distribution can be written as

$\widehat{A}_{x}(w)=1-w$

$$
+2 \sum_{i=1}^{n} \pi_{b, i}(x) \int_{0}^{w} B\left(u ; v W_{i} \theta_{b}(x)+\tau, v\left\{1-W_{i} \theta_{b}(x)\right\}+\tau\right) \mathrm{d} u,
$$

$$
\widehat{C}_{x}=2 \widehat{A}_{x}(1 / 2)=1
$$

$$
+4 \sum_{i=1}^{n} \pi_{b, i}(x) \int_{0}^{1 / 2} B\left(u ; v W_{i} \theta_{b}(x)+\tau, v\left\{1-W_{i} \theta_{b}(x)\right\}+\tau\right) \mathrm{d} u,
$$

$$
\begin{aligned}
\widehat{G}_{x}\left(y_{1}, y_{2}\right)= & \exp \left\{-2 \int_{0}^{1} \max \left(\frac{u}{y_{1}}, \frac{1-u}{y_{2}}\right)\right. \\
& \left.\times \sum_{i=1}^{n} \pi_{b, i}(x) \beta\left(u ; \nu W_{i} \theta_{b}(x)+\tau, v\left\{1-W_{i} \theta_{b}(x)\right\}+\tau\right) \mathrm{d} u\right\},
\end{aligned}
$$

for $x \in \mathcal{X}$, and $y_{1}, y_{2}>0$.

3.3. Connections to smoothing on the unit interval. Kernel density estimation on the unit interval is a challenging problem; see Chen (1999), Jones and Henderson (2007), de Carvalho et al. (2013), Geenens (2014), and the references therein. In this section, we contrast a stationary version of our estimator (3.2) with that of Chen (1999), and comment on the connections with the smooth Euclidean likelihood angular density of de Carvalho et al. (2013). The latter can be regarded as a moment constrained kernel density estimator on the unit interval, in the sense that it obeys (2.3).

If all covariates $x$ take the same value, so that the estimation problem reduces to one of estimating the angular density for an identically distributed set of pseudoangles $\left\{W_{i}\right\}_{i=1}^{n}$, then (3.2) becomes

$$
\widehat{h}(w)=\frac{1}{n} \sum_{i=1}^{n} \beta\left(w ; v \frac{W_{i}}{2 \bar{W}}+\tau, v\left\{1-\frac{W_{i}}{2 \bar{W}}\right\}+\tau\right), \quad w \in(0,1) .
$$

The version of our estimator in equation (3.4) differs from Chen's beta kernel [Chen (1999)]:

$$
h^{\star}(w)=\frac{1}{n} \sum_{i=1}^{n} \beta\left(W_{i} ; \frac{w}{s}+1, \frac{1-w}{s}+1\right)
$$


where $s>0$ is a bandwidth. Indeed, (3.5) puts the mode of the kernel at $W_{i}$ and so does our estimator in (3.4), if we set $\tau=1$. Yet, in (3.4) $w$ is the argument of $\beta(\cdot)$, whereas in (3.5), $W_{i}$ is the argument of $\beta(\cdot)$. Estimator (3.4) has closer connections with the smooth Euclidean angular density estimator in de Carvalho et al. (2013), page 1190, and which is given by

$$
\begin{aligned}
\tilde{h}(w)= & \frac{1}{n} \sum_{i=1}^{n}\left\{1-(\bar{W}-1 / 2) S^{-2}\left(W_{i}-\bar{W}\right)\right\} \beta\left\{w ; v W_{i}, v\left(1-W_{i}\right)\right\} \\
= & \frac{1}{n} \sum_{i=1}^{n} \beta\left\{w ; v W_{i}, v\left(1-W_{i}\right)\right\} \\
& -\frac{1}{n} \sum_{i=1}^{n}(\bar{W}-1 / 2) S^{-2}\left(W_{i}-\bar{W}\right) \beta\left\{w ; v W_{i}, v\left(1-W_{i}\right)\right\},
\end{aligned}
$$

for $w \in(0,1)$; here, $\bar{W}$ and $S^{2}$ are the sample mean and sample variance of $W_{1}, \ldots, W_{n}$, that is,

$$
\bar{W}=\frac{1}{n} \sum_{i=1}^{n} W_{i}, \quad S^{2}=\frac{1}{n} \sum_{i=1}^{n}\left(W_{i}-\bar{W}\right)^{2} .
$$

A heuristic argument can be used to see this, by focusing on the case $\tau=0$. The right-hand term in (3.6) enforces the moment constraint, and hence it is asymptotically negligible, so that for large $n$, we have $\widetilde{h}(w) \approx(1 / n) \sum_{i=1}^{n} \beta\{w$; $\left.v W_{i}, v\left(1-W_{i}\right)\right\}$; on the other hand, we also have that for large $n, \widehat{h}(w) \approx$ $(1 / n) \sum_{i=1}^{n} \beta\left\{w ; v W_{i}, v\left(1-W_{i}\right)\right\}$, since $\bar{W}=1 / 2+o_{\mathrm{p}}(1)$, as $n \rightarrow \infty$. While both (3.4) and (3.6) obey the moment constraint (2.6), they impose it through different approaches: our estimator enforces $(2.3)$ by rescaling the pseudo-angles with a factor of $(2 \bar{W})^{-1}$; the smooth Euclidean angular density enforces (2.3) additively, through the right-hand term in (3.6). To our knowledge, it is not straightforward to impose the moment constraint on Chen's kernel in (3.5).

3.4. Tuning parameter selection and bootstrap. We select the tuning parameters via maximum likelihood $K$-fold cross-validation (MLCV) [Hastie, Tibshirani and Friedman (2001), Section 7.10.1]. Specifically, let $\left\{\mathbf{W}_{1}, \ldots, \mathbf{W}_{K}\right\}$ be the full sample of pseudo-angles split into $K$ blocks. In the analyses in Sections 4 and 5, we split the blocks according to the values of the accompanying covariate $x$, so that each $\mathbf{W}_{k}=\left(W_{k, 1}, \ldots, W_{k, n_{k}}\right)$ is in a similar part of the covariate space. Letting $\widehat{h}_{x(-k)}$ denote the estimator leaving out the $k$ th sample, $\mathbf{W}_{k}$, of length $n_{k}$, we select

$$
(\widehat{b}, \widehat{v}, \widehat{\tau})=\arg \min _{(b, \nu, \tau) \in \mathcal{R}_{\mathcal{X}, n}} \sum_{k=1}^{K} \sum_{j=1}^{n_{k}}-\log \widehat{h}_{X_{k, j}(-k)}\left(W_{k, j}\right),
$$


with

$$
\begin{aligned}
\mathcal{R}_{\mathcal{X}, n}= & \left\{(b, v, \tau) \in(0, \infty)^{3}: v W_{i} \theta_{b}(x)+\tau>0,\right. \\
& \left.v\left\{1-W_{i} \theta_{b}(x)\right\}+\tau>0, \text { for } i=1, \ldots, n ; x \in \mathcal{X}\right\} \\
= & \left\{(b, v, \tau) \in(0, \infty)^{3}: v\left\{1-W_{i} \theta_{b}(x)\right\}+\tau>0, \text { for } i=1, \ldots, n ; x \in \mathcal{X}\right\} .
\end{aligned}
$$

The constrained optimization yields well-defined estimates, since it guarantees the positivity of the beta parameters in our estimator. The latter equality in (3.8) follows from noticing that $v W_{i} \theta_{b}(x)+\tau>0$, for all $x \in \mathcal{X}$; further details on practical implementation of tuning parameter selection are given in Section 4.2. It is known that for density estimation, MLCV can produce estimates with suboptimal performance leading to undersmoothed density estimates, especially when the true density has unbounded support [DasGupta (2008), Section 32.10.1]. Computational experiments in the supplementary materials [Castro-Camilo, de Carvalho and Wadsworth (2018)] show that the main findings in Section 5 are very similar regardless of whether we use MLCV or least-squares cross-validation (LSCV) [DasGupta (2008), Section 32.10.2]. Better results than the ones in Section 4 are to be expected if LSCV is used. However, LSCV would not be theoretically grounded for nonsquare integrable densities [e.g., $h_{x}(w)=\beta(w ; x, x)$, for $x \in(0,1 / 2)$ ].

An uncertainty assessment can be performed by simulating from kernel density estimates themselves - in the spirit of the so-called smoothed bootstrap [Silverman and Young (1987)]. The procedure detailed below, allows us to generate $B$ bootstrap angular surfaces. For $r \in\{1, \ldots, B\}$ :

1. Sample $j^{\star}$ from a discrete uniform distribution over $\{1, \ldots, n\}$.

2. Sample $X_{j}^{r} \sim K_{\widehat{b}}\left(\cdot-X_{j^{\star}}\right)$.

3. Sample $W_{j}^{r} \sim \widehat{h}_{X_{j}^{r}}$ with

$$
\widehat{h}_{X_{j}^{r}}(w)=\sum_{i=1}^{n} \pi_{\widehat{b}, i}\left(X_{j}^{r}\right) \beta\left(w ; \widehat{v} W_{i} \theta_{\widehat{b}}\left(X_{j}^{r}\right)+\widehat{\tau}, \widehat{v}\left\{1-W_{i} \theta_{\widehat{b}}\left(X_{j}^{r}\right)\right\}+\widehat{\tau}\right),
$$

for $w \in(0,1)$, and where for $i=1, \ldots, n$ :

$$
\theta_{\widehat{b}}\left(X_{j}^{r}\right)=\frac{1 / 2}{\sum_{i=1}^{n} \pi_{\widehat{b}, i}\left(X_{j}^{r}\right) W_{i}}, \quad \pi_{\widehat{b}, i}\left(X_{j}^{r}\right)=\frac{K_{\widehat{b}}\left(X_{j}^{r}-X_{i}\right)}{\sum_{k=1}^{n} K_{\widehat{b}}\left(X_{j}^{r}-X_{k}\right)} .
$$

4. Repeat Steps 1-3 $n$ times to obtain the $r$ th bootstrap sample $\left(\mathbf{X}^{r}, \mathbf{W}^{r}\right)$, with

$$
\mathbf{X}^{r}=\left(X_{1}^{r}, \ldots, X_{n}^{r}\right)^{\mathrm{T}}, \quad \mathbf{W}^{r}=\left(W_{1}^{r}, \ldots, W_{n}^{r}\right)^{\mathrm{T}} .
$$

5. Use $\left(\mathbf{X}^{r}, \mathbf{W}^{r}\right)$ and (3.7) to obtain bootstrap estimates $\left(\widehat{b}^{r}, \widehat{v}^{r}, \widehat{\tau}^{r}\right)$.

Using the bootstrap samples $\left\{\left(\mathbf{X}^{r}, \mathbf{W}^{r}\right)\right\}_{r=1}^{B}$, the bootstrap estimates $\left\{\left(\widehat{b}^{r}, \widehat{v}^{r}\right.\right.$, $\left.\left.\widehat{\tau}^{r}\right)\right\}_{r=1}^{B}$, and (3.2), we can construct $B$ bootstrap angular surfaces $\widehat{h}_{x}^{1}, \ldots, \widehat{h}_{x}^{B}$. For computational convenience, Step 2 considers only a single bandwidth, $\widehat{b}$, but it is 
known [see, e.g., Polansky (2001)] that smoothed bootstrap resamples need not be generated from the kernel density estimate with the same bandwidth. Indeed, the so-called calibration methods are known to perform well, but they require one to construct a resample over a sequence of bandwidths, and thus are computationally costlier. Visualizing uncertainty of angular surfaces can be awkward, but cross sections of the angular surface (i.e., conditional angular density estimates at fixed values of $x$ ) can be easily summarized using, for example, functional boxplots [Sun and Genton (2011)]. Details on constructing functional boxplots for angular densities are given in Section 4.2.

3.5. A local-linear version of the estimator. A local linear version of our estimator can be readily constructed by replacing the Nadaraya-Watson weights in (3.2) with

$$
\pi_{b, i}(x)=\frac{1}{n} \frac{\left\{\widehat{s}_{2}(x ; b)-\widehat{s}_{1}(x ; b)\left(X_{i}-x\right)\right\} K_{b}\left(X_{i}-x\right)}{\widehat{s}_{2}(x ; b) \widehat{s}_{0}(x ; b)-\widehat{s}_{1}^{2}(x ; b)},
$$

where $\widehat{s}_{m}(x ; b)=n^{-1} \sum_{i=1}^{n}\left(X_{i}-x\right)^{m} K_{b}\left(X_{i}-x\right)$, for $m=0,1,2$. Local linear regression is often presented as a solution to mitigate boundary bias issues of the Nadaraya-Watson estimator [Wand and Jones (1995), Section 5.5]. Throughout, we consider both Nadaraya-Watson and local linear weights to illustrate their relative performance.

\section{Simulation study.}

4.1. Data-generating configurations and preliminary experiments. We study the performance of our methods under the logistic and Dirichlet conditional models introduced in Examples 1 and 2. Regarding the logistic conditional model, we take $\alpha_{x}=\Phi(x)$ and consider $x \in \mathcal{X}_{\text {logistic }}=\left[\Phi^{-1}(0.2), \Phi^{-1}(0.4)\right]$. For the Dirichlet conditional model, we consider two scenarios: a symmetric Dirichlet angular surface with $\left(a_{x}, b_{x}\right)=(x, x)$, for $x \in \mathcal{X}_{\mathrm{sDir}}=[0.8,4]$ and an asymmetric Dirichlet angular surface with $\left(a_{x}, b_{x}\right)=(x, 100)$, for $x \in \mathcal{X}_{\mathrm{aDir}}=[0.5,2]$. In Figure 2, we plot the true and estimated angular surfaces for the three cases described above on a single experiment with $n=500$. The top panel of Figure 2 corresponds to the logistic angular surface, where extremal dependence decreases as a function of the predictor. The center panel shows the symmetric Dirichlet angular surface, where we observe weaker dependence for lower values of the covariate, whereas stronger dependence prevails for higher values. Finally, an increasing asymmetric dependence dynamic is displayed in the bottom panel, where we have plotted the asymmetric Dirichlet angular surface.

The single run experiment in Figure 2 allows us to illustrate strengths and limitations with the methods. Even though there is a good fit-which is discussed in further detail in Section 4.2-we can anticipate from this figure that our estimator suffers from limitations inherent to kernel-based estimators. For example, 
Logistic angular surface
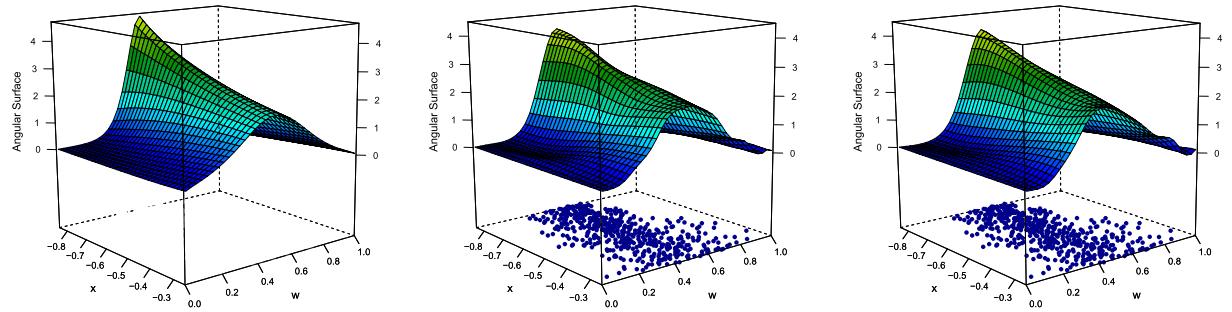

Symmetric Dirichlet angular surface
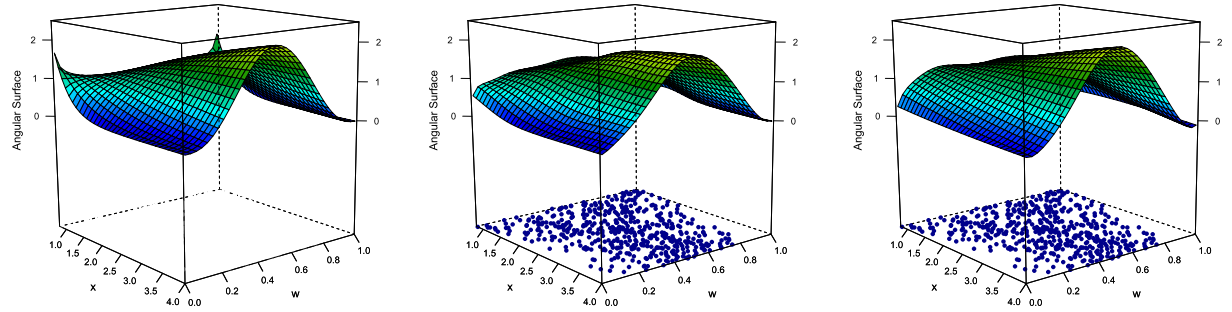

Asymmetric Dirichlet angular surface
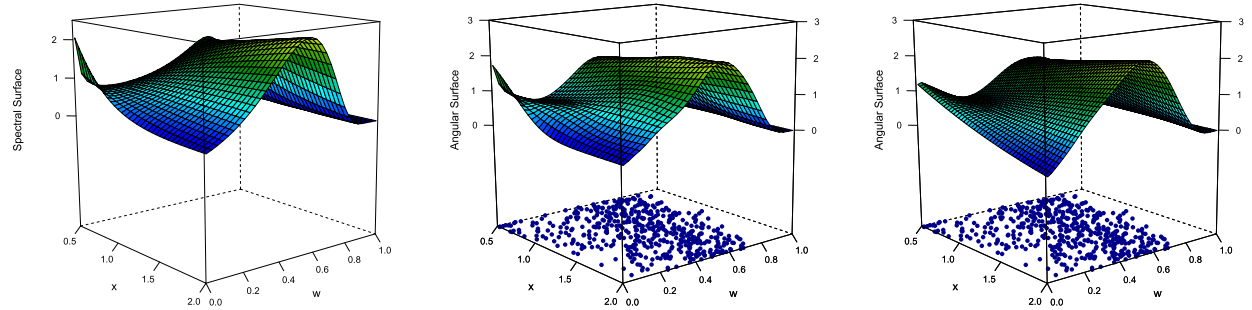

FIG. 2. True angular surfaces (left) and corresponding estimates using Nadaraya-Watson weights (middle) and local linear weights (right). Top panel: conditional logistic model with $\alpha_{x}=\Phi(x)$, for $x \in \mathcal{X}_{\text {logistic }}=\left[\Phi^{-1}(0.2), \Phi^{-1}(0.4)\right]$. Center panel: conditional Symmetric Dirichlet model with $\left(a_{x}, b_{x}\right)=(x, x)$, for $x \in \mathcal{X}_{\mathrm{sDir}}=[0.8,4]$. Bottom panel: conditional Asymmetric Dirichlet model with $\left(a_{x}, b_{x}\right)=(x, 100)$, for $x \in \mathcal{X}_{\mathrm{aDir}} \in[0.5,2]$. The simulated pseudo-angles based on which the estimates are produced are overlaid on the bottom of the boxes.

pointwise estimation using the Nadaraya-Watson weights (middle column of Figure 2) underperforms when the angular surface peaks, but this is mostly due to the boundary bias of $K_{b}$ which is a drawback of kernel-based estimators on bounded domains; see Härdle (1990), Section 4.4, and references therein. To mitigate this issue, we also compute our estimator using local linear weights, as described in Section 3.5 (right column of Figure 2). We see that the performance in the upper boundaries of the covariate space is slightly improved for both Dirichlet angular 
TABLE 1

Mean integrated absolute error estimates computed over 1000 samples for the data-generating configurations discussed in Section 4.1 for the Nadaraya-Watson $(N-W)$ and the local lineal $(L-L)$ weights

\begin{tabular}{llccc}
\hline & & & \multicolumn{2}{c}{ MIAE } \\
\cline { 4 - 5 }$n$ & Conditional model & Specification & N-W weights & L-L weights \\
\hline 300 & Logistic & $\alpha_{x}=\Phi(x)$ & 0.09 & 0.92 \\
& Symmetric Dirichlet & $\left(a_{x}, b_{x}\right)=(x, x)$ & 0.42 & 0.60 \\
& Asymmetric Dirichlet & $\left(a_{x}, b_{x}\right)=(x, 100)$ & 0.63 & 0.59 \\
500 & Logistic & $\alpha_{x}=\Phi(x)$ & 0.08 & 0.14 \\
& Symmetric Dirichlet & $\left(a_{x}, b_{x}\right)=(x, x)$ & 0.39 & 0.55 \\
& Asymmetric Dirichlet & $\left(a_{x}, b_{x}\right)=(x, 100)$ & 0.62 & 0.55 \\
\hline
\end{tabular}

surfaces, but it remains almost the same for the logistic angular surface. The estimator using local linear weights seems to produce smoother estimates for the asymmetric Dirichlet model. This relative improvement is corroborated in Table 1, where we assess the mean performance of both estimators. Estimates for the other two models tend to be better (in terms of mean performance) using the NadarayaWatson weights. In terms of computations, the runtime of the estimator using the Nadaraya-Watson weights outperforms its local linear counterpart by at least a factor of 10. In spite of these limitations, both estimators successfully recover the shape of the true angular surface, and thus are able to reproduce accurately the evolution of extremal dependence over the covariate.

4.2. Simulation results. To construct the simulation studies, we took 1000 samples of sizes 300 and 500 for the three conditional models presented in Section 4.1. For the samples of size 500, Figure 3 displays functional boxplots [Sun and Genton (2011)] of cross sections of the angular surface (conditional angular density estimates at fixed values of $x$ ) along with their Monte Carlo means. Functional boxplots are constructed introducing measures to define functional quantiles and the centrality or outlyingness of a curve. Specifically, Sun and Genton (2011) use band depths to order a sample of curves from the center outwards, defining $100 \alpha \%$ central regions $(0<\alpha<1)$. These central regions can be estimated using the $\alpha$ proportion of deepest curves; a formal definition of these regions can be found in Sun and Genton (2011), Section 3. The gray areas in Figure 3 show the sample 50\%, 75\%, and 95\% central regions of the sampled curves. These plots allow us to illustrate the performance of our estimator in terms of variability, under different dependence dynamics. For example, the logistic model estimates presented in the top panel of Figure 3 turn out to be the most dispersed over all three scenarios. We argue that there are two related reasons for this: the limitations due to boundary bias that were discussed in Section 4.1, and the fact that the range 


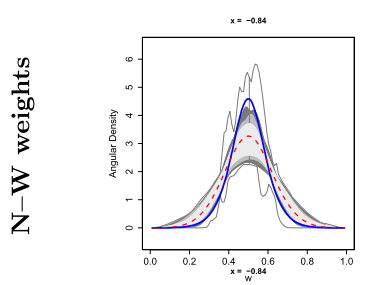

Logistic angular surface
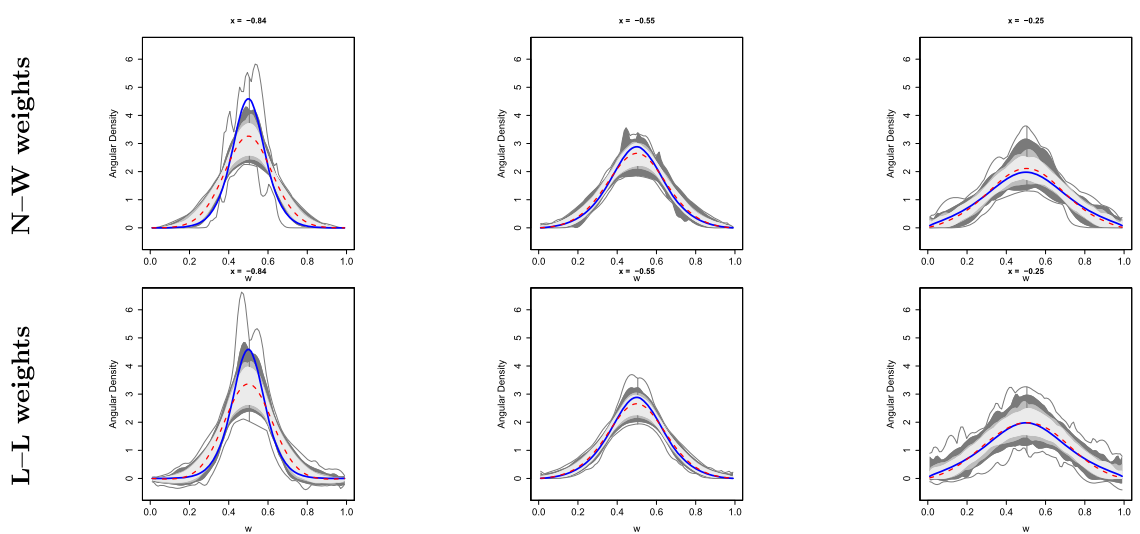

Symmetric Dirichlet angular surface
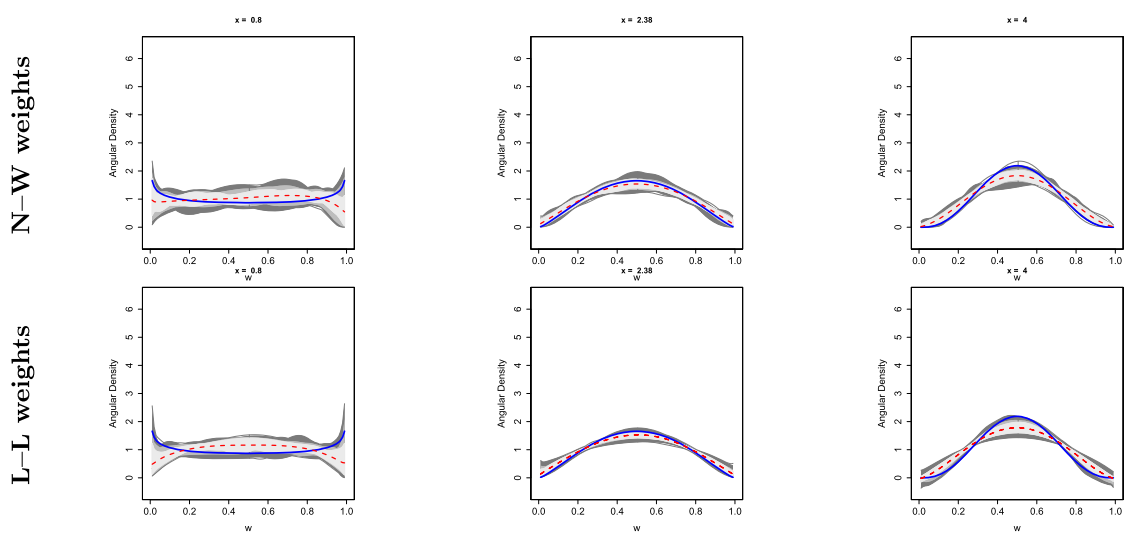

Asymmetric Dirichlet angular surface
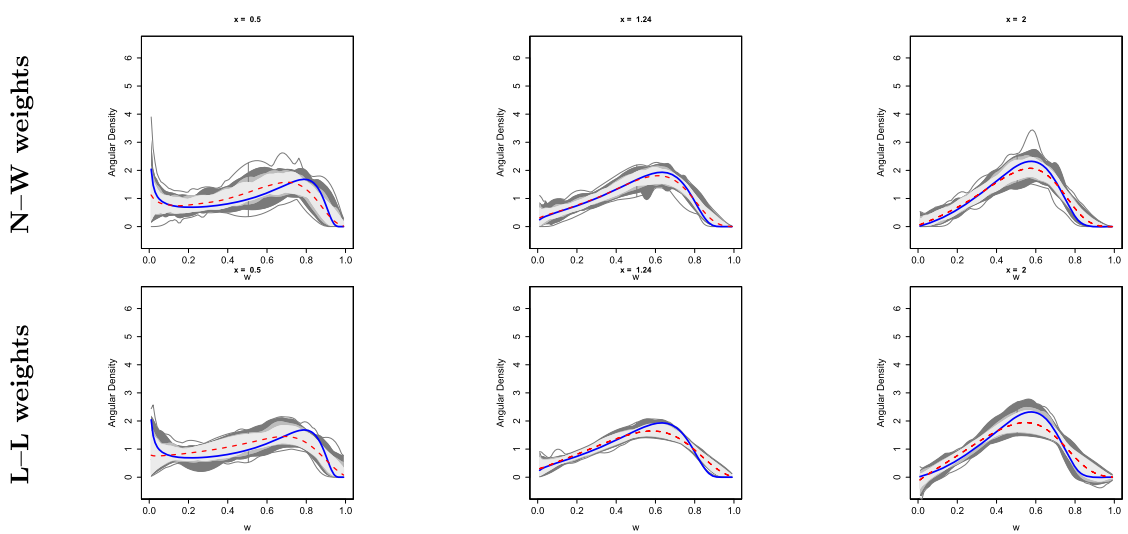

FIG. 3. Functional boxplots (gray shadow) showing the 50\%, 75\%, and 95\% central regions [as defined by Sun and Genton (2011)] of 1000 samples of size 500 for the conditional models presented in Section 4.1, as well as their corresponding true values (solid blue line) and Monte Carlo means (dashed red line). 
of extremal dependence in the logistic conditional surface is greater compared to the two other scenarios. Both estimators seems to perform similarly, although the local-linear estimator is more variable when the extremal dependence is stronger. The center panel corresponds to the symmetric Dirichlet angular model, which displays a good mean performance in the last two cases, but some bias when the true angular density is U-shaped. Estimates using the Nadaraya-Watson weights seem to be slightly more variable than the ones using the local linear weights. Finally, the asymmetric Dirichlet angular model presented in the bottom panel, displays more dispersed estimates than its symmetric counterpart for both estimators (and between them it seems that the Nadaraya-Watson estimates are again more variable than the ones using local linear weights), although the Monte Carlo mean produces suitable approximations. The asymmetry does not seem to be a major issue. Overall, estimates for the three models display reasonable performance in recovering the different shapes of the densities, and Monte Carlo means produce reliable estimates. Monte Carlo mean surfaces for the three models and the two estimators can be found in the supplementary material.

We assess the performance of our estimator using the mean integrated absolute error (MIAE),

$$
\operatorname{MIAE}=\mathrm{E}\left(\int_{\mathcal{X}} \int_{0}^{1}\left|\widehat{h}_{x}(w)-h_{x}(w)\right| \mathrm{d} w \mathrm{~d} x\right),
$$

and report the results in Table 1. As mentioned before, we can see that the estimator using the Nadaraya-Watson weights outperforms the one using local linear weights in the logistic and symmetric Dirichlet models, but the local linear weights seem to be a better choice for the asymmetric Dirichlet model. In any case and except for the logistic model with sample size 300, the improvements of one estimator over the other are fairly modest. As we should expect, the results show that performance increases with sample size. Overall, simulations confirm that our methods produce acceptably accurate estimates of the angular surface.

We conclude this section providing some comments on implementation of the tuning parameter selection [Section (3.4)]. Since in some cases optimization over $\mathcal{R}_{\mathcal{X}, n}$ [defined in equation (3.8)] can be computationally expensive, our experiments suggest that optimization over $\mathcal{R}_{n}$ defined as

$$
\mathcal{R}_{n}=\left\{(b, v, \tau) \in(0, \infty)^{3}: v\left\{1-W_{i} \theta_{b}\left(X_{j}\right)\right\}+\tau>0, \text { for } i, j=1, \ldots, n\right\},
$$

performs reasonably well. Note that $\mathcal{R}_{n}$ is a version of $\mathcal{R}_{\mathcal{X}, n}$ determined only by the observed covariate values, and not by the entire covariate space $\mathcal{X}$. Furthermore, for large $n$, unconstrained optimization over $(0, \infty)^{3}$ typically also performs well. We thus recommend the user to initially try unconstrained optimization for large $n$, or optimization over $\mathcal{R}_{n}$ for moderate $n$. Only if the resulting parameter values do not yield a valid estimator over the study region of interest does one then need to implement the constrained optimization over $\mathcal{R}_{\mathcal{X}, n}$. 


\section{Dynamics of joint extremal losses in leading European stock markets.}

5.1. Background and motivation for empirical analysis. In 1999, 11 European Union (EU) countries formed the Economic and Monetary Union (EMU), which led them to adopt a common currency and monetary policy as well as the conduction of coordinated economic policies.

The process of creation of the EMU was the outcome of three stages of development, further details of which can be found on the European Central Bank website:

\section{https://www.ecb.europa.eu/ecb/history}

See also James (2012). To join the Eurozone (countries who adopted the Euro as their common currency) member states had to qualify by meeting the criteria of the Maastricht Treaty in terms of budget deficits, inflation, interest rates, and other monetary requirements. At the moment the Euro is the single currency shared by 19 of the 28 EU members. The remaining 9 countries, including the UK, are endowed with "opt-out" clauses which exempts them from using the Euro as their currency. In recent years, there have been several studies providing evidence for an increased integration of European stock markets, and the EMU has been frequently put forward as the causal driver for this increase, along with some other determinants [Büttner and Hayo (2011), Fratzscher (2002), Kim, Moshirian and Wu (2005), Hardouvelis, Malliaropulos and Priestley (2006), and the references therein]. Hardouvelis, Malliaropulos and Priestley (2006) found however that the UK, who chose not to enter the eurozone, showed no increase in stock market integration by that time.

Although there is a wealth of studies analyzing stock market integration over time, few attempts have been made to ascertain the dynamics governing extreme value dependence of stock market returns over time. The huge literature looking into dependence of financial markets [see, e.g., Forbes and Rigobon (2002), Brooks and Del Negro (2004, 2005), Karolyi and Stulz (1996), King, Sentana and Sushil (1994), Longin and Solnik (1995, 2001), Rua and Nunes (2009)] has collected evidence compatible with the hypothesis that the comovement of returns has not remained constant over time. Yet, none of these papers has focused on tracking the dynamics of extremal dependence of returns, which is the object of the current inquiry. An exception in this respect is the seminal paper of Poon, Rockinger and Tawn (2003), which provides evidence of increasing levels of extremal dependence for three major stock markets within Europe [CAC (France), DAX (Germany), and FTSE (UK)]. The subperiod analysis of Poon, Rockinger and Tawn (2003), Section 3.3.2, is however exploratory, in the sense that they arbitrarily partitioned the sample period into three periods, and thus estimation of extremal dependence on each period only takes data from that period into account. 
Below, we apply our methods to address a similar question to that of Poon, Rockinger and Tawn (2003, 2004). Specifically, one of our main interests is disentangling the dynamics governing the dependence of extreme losses on three leading European stock markets - using CAC, DAX, and FTSE_-in recent years. The motivation for choosing these markets is twofold: these are the stock markets of the European members of G5; these are also the same European stock markets considered by Poon, Rockinger and Tawn (2003, 2004). Moreover, they display a stronger type of extremal dependence than some of the other markets studied by Poon, Rockinger and Tawn (2003, 2004), that is, asymptotic dependence as defined in Section 2.1.

5.2. Data description, preprocessing, and exploratory considerations. Our data were gathered from Datastream and consist of daily closing stock index levels of three leading European stock markets: CAC 40, DAX 30, and FTSE 100 (henceforth CAC, DAX, and FTSE). The sample period spans from January 1, 1988, to January 1,2014 ( $N=6784$ observations), and hence it includes the Great Moderation and Great Recession which are by all standards challenging modeling issues. Since we want to focus on extreme losses, we use daily negative returns as a unit of analysis. Daily negative returns are computed by taking the negative of the first differences of the logarithmic indices. Following the bivariate analysis in Poon, Rockinger and Tawn (2004), both observations of a particular day are removed if at least one of the two observations is a zero return (plots of the data and summary statistics can be found in the supplementary material). The Engle (1) statistic of Engle (1982) (not reported here) is large and significant for all three stock return series, indicating strong heteroskedasticity which can be removed by fitting volatility filters. In the spirit of Poon, Rockinger and Tawn (2004), we fit three different filters: $\operatorname{GARCH}(1,1)$ assuming $t$-distributed errors for $\mathrm{CAC}$ and normal for FTSE and DAX, NGARCH (also known as nonlinear asymmetric GARCH) with normal innovations, and the stochastic volatility model (SV) of Kim, Shephard and Chib (1998) with hyperparameters chosen according to the latter paper. Diagnostic plots (not shown here) suggest that the GARCH fits are superior than the NGARCH fits for the three stock markets, and heteroskedasticity is successfully removed with the GARCH and NGARCH filters, but not with the SV filter. The results shown below correspond to the GARCH-filtered residuals, but similar conclusions can be drawn using the NGARCH filter (angular surfaces based on the NGARCH-filtered residuals can be found in the supplementary material). Scatterplots of possible combinations of pairs of filtered residual series are displayed in Figure 4, depicted using a time-varying color palette which allows us to uncover the nonstationary nature of joint extremes. This is in line with the findings of Poon, Rockinger and Tawn (2003, 2004).

To verify that our methods are a sensible approach for modeling these data, we need to assess whether the filtered residuals are asymptotically dependent. As mentioned in Section 2.1, in the modeling of extreme events two different classes 

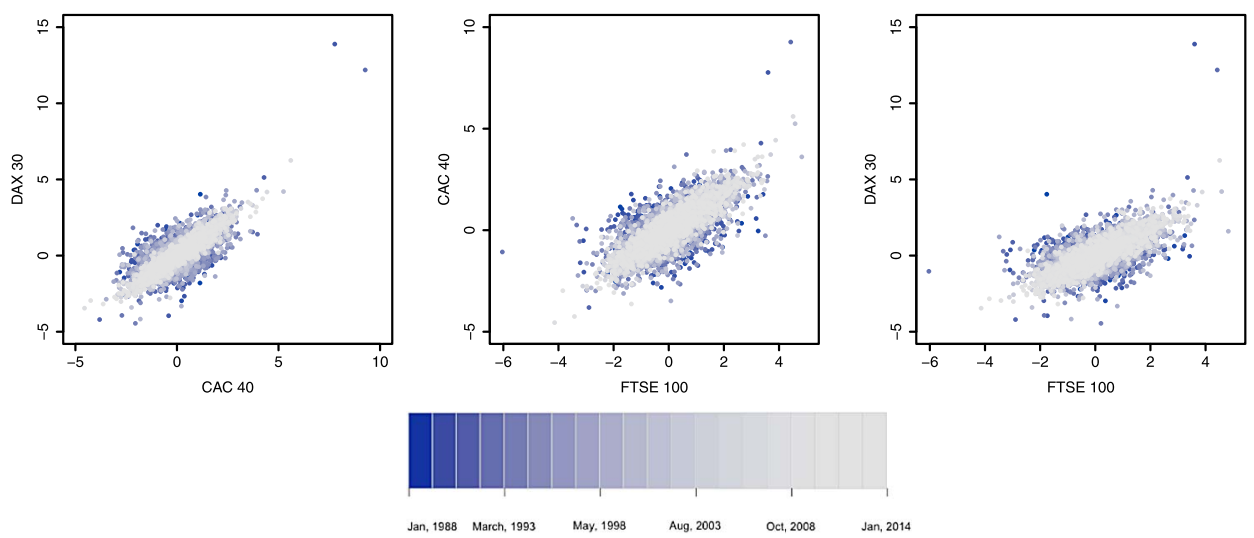

FIG. 4. Scatterplots using a time-varying color palette for GARCH-filtered residuals for CAC 40 (FR), DAX 30 (DE) and FTSE 100 (UK) spanning the period from January 1, 1988, to January 1, 2014.

of extreme value dependence can arise: asymptotic dependence and asymptotic independence. Dependence between moderately large values can arise in both cases, but the very largest values from each variable can occur together only under asymptotic dependence. To make ideas concrete, let $Y_{1}$ and $Y_{2}$ be any two filtered residuals of interest, transformed to have unit Fréchet margins. Under an exploratory setting, two measures of tail dependence can be obtained to summarize the strength of extremal dependence:

$$
\chi=\lim _{u \rightarrow \infty} \mathrm{P}\left(Y_{1}>u \mid Y_{2}>u\right), \quad \bar{\chi}=\lim _{u \rightarrow \infty} \frac{2 \log \mathrm{P}\left(Y_{1}>u\right)}{\log \mathrm{P}\left(Y_{1}>u, Y_{2}>u\right)}-1 .
$$

Here, $\chi \in[0,1]$ measures the strength of dependence within the class of asymptotically dependent variables, whereas $\bar{\chi} \in[-1,1]$ is often used to measure the strength of dependence within the class of asymptotically independent variables. Taken together, the pair $(\chi, \bar{\chi})$ provides a summary of extremal dependence for the vector $\left(Y_{1}, Y_{2}\right)$. For asymptotically dependent variables, we have $\bar{\chi}=1$ and the value of $\chi>0$ increases with the strength of dependence at extreme levels. For asymptotically independent variables, we have $\chi=0$ and $\bar{\chi} \leq 1$ increases with the strength of dependence at extreme levels. Roughly speaking, if $\bar{\chi}>0$ then we often speak about "positive extremal dependence", whereas if $\bar{\chi}<0$ we use the expression "negative extremal dependence". Indeed, for the bivariate normal dependence structure $\bar{\chi}$ corresponds to Pearson correlation; see Heffernan (2000) for further examples.

In Figure 5, we present rolling window estimates of $\chi$ and $\bar{\chi}$ with approximate $95 \%$ confidence intervals, which is tantamount to the subperiod analysis of Poon, Rockinger and Tawn (2003), Section 3.3.2. The rolling window estimates were computed using the empirical estimators of $\chi$ and $\bar{\chi}$ [Beirlant et al. (2004), 

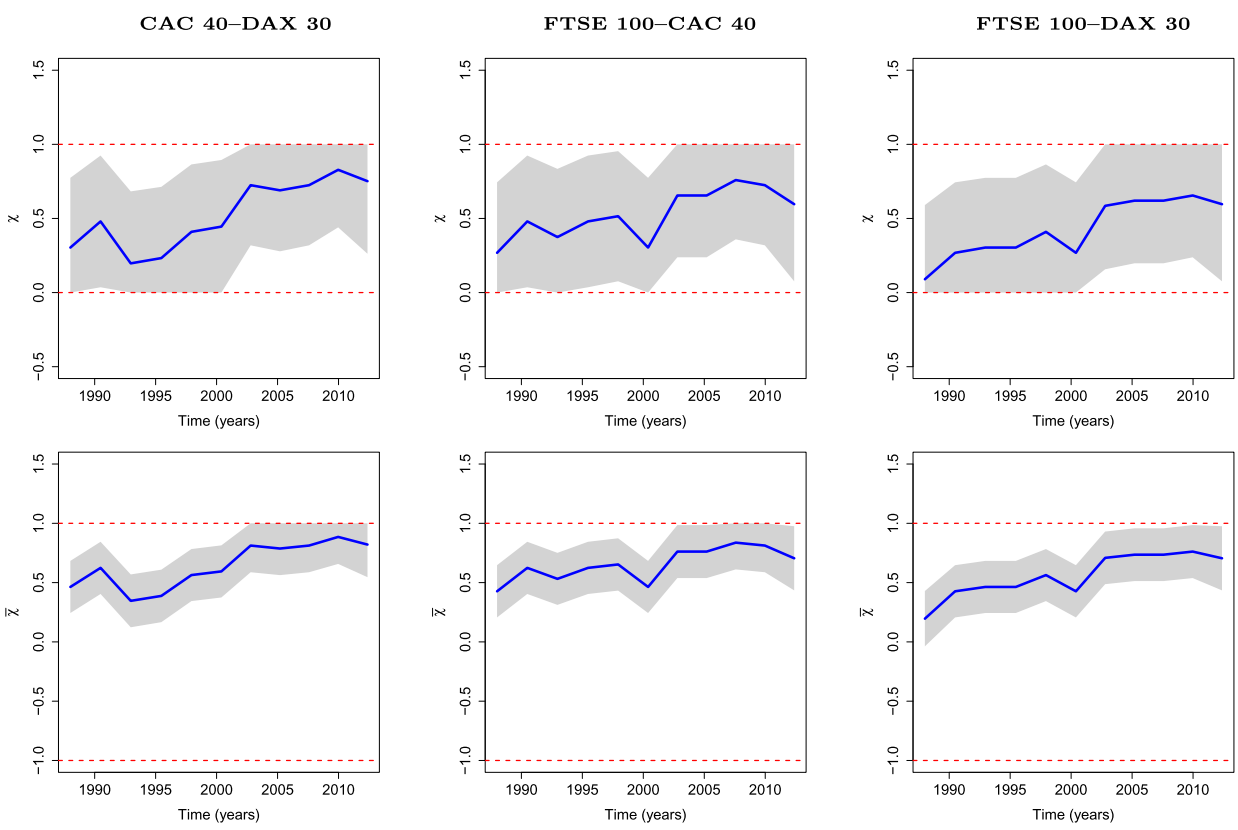

FIG. 5. Rolling window estimates of $\chi$ (top) and $\bar{\chi}$ (bottom) at the $95 \%$ quantile using moving windows of size 600, applied to the three pairs under study.

page 348] at the $95 \%$ quantile for moving windows of 600 observations. Given the large uncertainty entailed in the estimation of $\chi$, interpretation of these plots is far from straightforward. Nevertheless, pointwise estimation for $\chi$ seems reasonably different from 0 for the three pairs under study, and despite some drops around 1992 and 2000, there seems to be an increasing trend for the three cases. Moreover, values for $\bar{\chi}$ are closer to 1 as time passes. This combined information indicates that the assumption of asymptotic dependence is certainly plausible for the later years, and might be adequate for earlier years. We discuss the asymptotic independence issue again in Section 6.

5.3. Modeling time-varying extremal dependence. The time-varying color palette scatterplots in Figure 4 and the rolling window estimates in Figure 5 provide evidence of nonstationary extremal dependence, but they are only exploratory. In this section, we complete the analysis from Section 5.2 by applying our conditional modeling approach to assess how the dependence structure of bivariate extreme losses in the three pairs has been evolving over recent years. Before we proceed any further, some comments regarding implementation are in order. As mentioned in Section 2, the data were transformed to have standard Fréchet margins. This was done as follows. Given a sample of pairs of filtered residuals $\left(r_{1,1}, r_{1,2}\right), \ldots,\left(r_{N, 1}, r_{N, 2}\right)$, we construct proxies for the unobservable pseudo- 
angles $W_{i}$ by setting

$$
W_{i}=\widehat{Y}_{i, 1} /\left(\widehat{Y}_{i, 1}+\widehat{Y}_{i, 2}\right), \quad R_{i}=\widehat{Y}_{i, 1}+\widehat{Y}_{i, 2}
$$

where $\widehat{Y}_{i, 1}=-1 / \log \left\{\widehat{F}_{r_{1}}\left(r_{i, 1}\right)\right\}$ and $\widehat{Y}_{i, 2}=-1 / \log \left\{\widehat{F}_{r_{2}}\left(r_{i, 2}\right)\right\}$ and where $\widehat{F}_{r_{1}}$ and $\widehat{F}_{r_{2}}$ are estimates of the marginal distribution functions $F_{r_{1}}$ and $F_{r_{2}}$. A robust choice for $\widehat{F}_{r_{1}}$ and $\widehat{F}_{r_{2}}$ is the pair of univariate empirical distribution functions, normalized by $N+1$ rather than by $N$ to avoid division by zero. Following Section 3.1, after fitting a spline-based nonparametric quantile regression we found evidence of dependence of the pseudo-radii $\left\{R_{1}, \ldots, R_{N}\right\}$ on time, and so we proceed under a nonstationary assumption. Specifically, we model the $95 \%$ quantile of the pseudo-radii through nonparametric quantile regression and threshold the pseudo-radii according to the fit. The tail region to study the extreme losses is therefore defined through the pseudo-angles associated with the threshold exceedances of the pseudo-radii. After thresholding, the number of pseudo-angles is 312 for CAC-DAX and FTSE-CAC and 314 for FTSE-DAX. The pseudo-angles corresponding to these observations are plotted in the two-dimensional bottom plane in Figure 7 . The tuning parameters $(b, v, \tau)$ were computed as discussed in Sections 3.4 and 4.2 .

In Figure 6, we plot cross sections of the angular surface estimate, using both Nadaraya-Watson and local linear weights as described in Section 3, at three important periods on the EU agenda: (I) Beginning of stage one of EMU (1 July, 1990); (II) beginning of stage three of EMU (1 January, 1999); (III) activation of the assistance package for Greece (2 May, 2010), the first country to be shut out of the bond market, which fostered the European sovereign debt crisis [Lane (2012)]. The choice of landmarks (I)-(III) is arbitrary, but recall that our main interest is in describing how extremal dependence may change, by comparing periods sufficiently apart in time. As can be observed from the first column in Figure 6, at around 1990 the dependence between extreme losses for the three pairs were similar, exhibiting some evidence of extremal independence, that is also reflected in Figure 5. The second column in Figure 6 reveals that about a decade later this dynamic changed, and that extreme losses started to show some mild signs of extremal dependence. These signs become stronger, and 11 years later (third column in Figure 6) we can clearly see evidence of extremal dependence of joint losses. Our findings may seem to contradict Hardouvelis, Malliaropulos and Priestley (2006) — who claimed that the UK showed no increase in stock market integration-however we note that Hardouvelis, Malliaropulos and Priestley (2006) did not assess extremal dependence. The functional boxplots in Figure 6 were obtained following the bootstrap procedure detailed in Section 3.4, with $B=1000$ samples. We can clearly see some differences between the two types of estimators among the three pairs, but overall they report similar information in terms of the extremal dependence. 


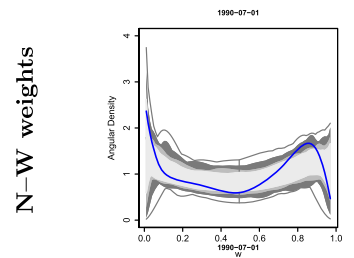

CAC 40-DAX 30
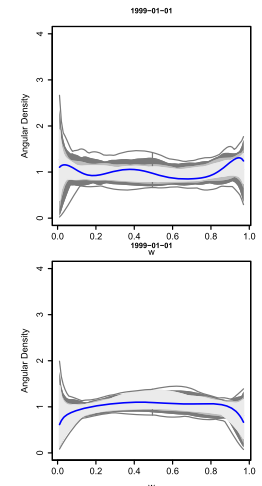

FTSE 100-CAC 40
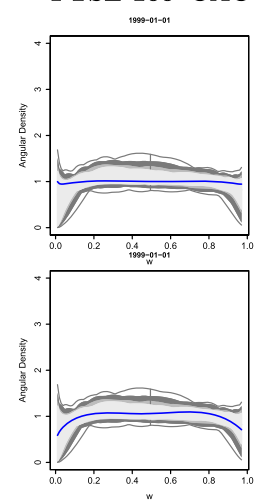

FTSE 100-DAX 30
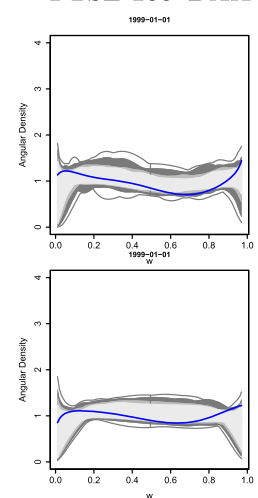
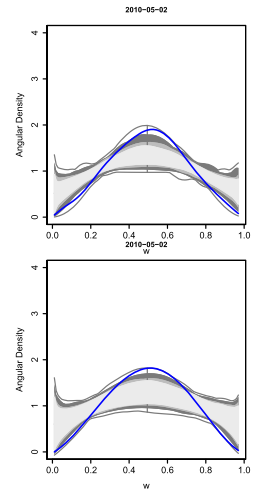

$2010-0-5-02$
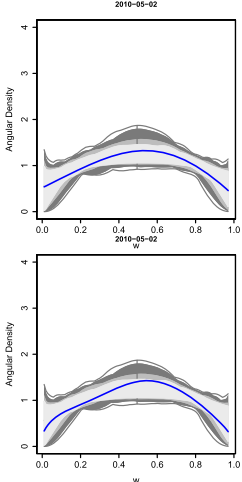

2010-05-00
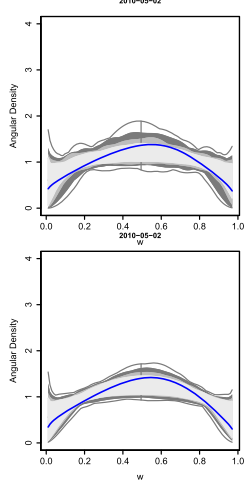

FIG. 6. Cross sections of angular surface estimates for CAC-DAX (top), FTSE-CAC (center), and FTSE-DAX (bottom) for Nadaraya-Watson and local linear weights (solid blue lines). The first column corresponds to the beginning of stage one of EMU (1 July 1990), the second column corresponds to the beginning of stage three of EMU (1 January 1999), and the third column corresponds to the time of activation of the assistance package for Greece (2 May 2010). Functional boxplots (gray shadows) show the 50\%, 75\%, and 95\% central regions [as defined by Sun and Genton (2011)] based on 1000 bootstrap samples. 

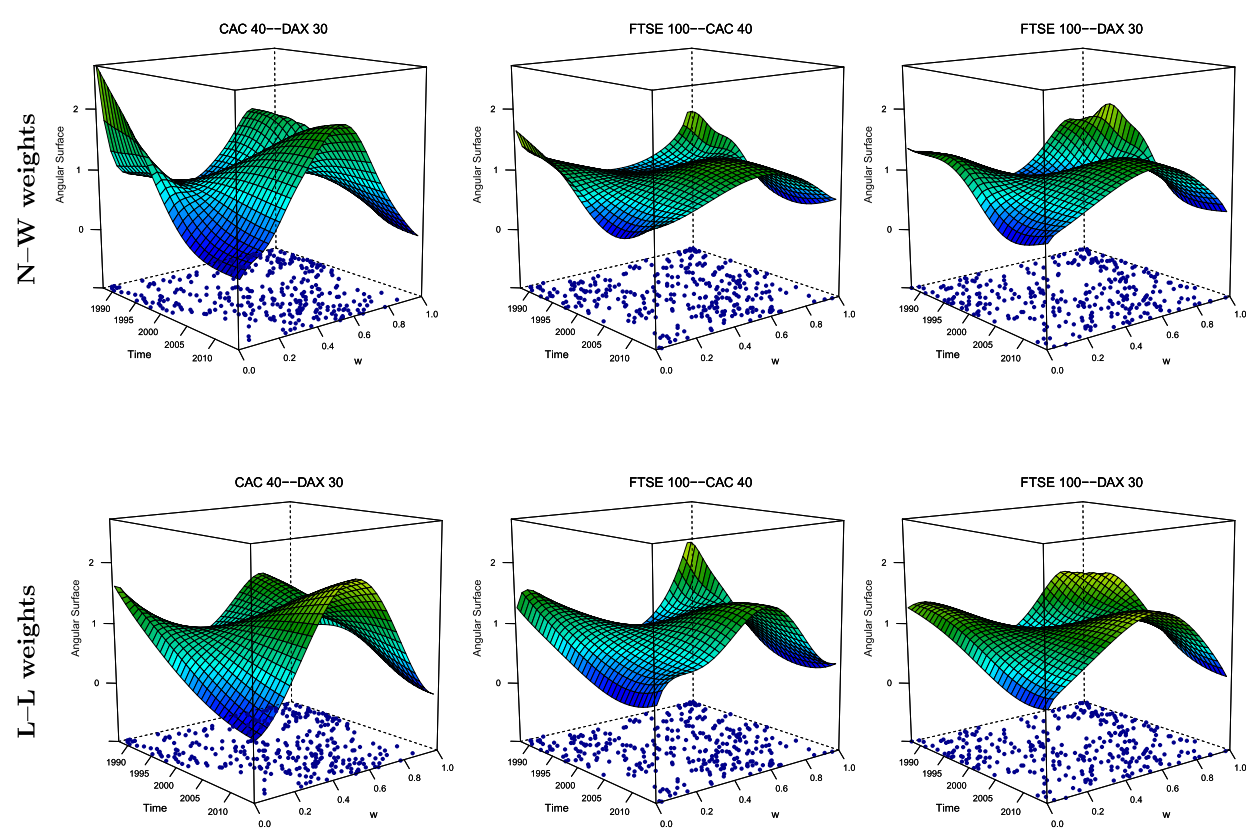

FIG. 7. Angular surface estimates for CAC-DAX, FTSE-CAC and FTSE-DAX using Nadaraya-Watson (top) and local linear (bottom) weights, with pseudo-angles overlaid on the bottom of the box.

Figure 6 provides only a few snapshots corresponding to landmarks (I)-(III). A more complete portrait of the temporal changes in extremal dependence is provided by the angular surface estimate in Figure 7, from which the cross-sections in Figure 6 are derived.

All in all, we can clearly see the change from weaker dependence around 1990 to strong dependence starting from 2005, thus suggesting that in recent decades there has been an increase in the extremal dependence in the losses for these leading European stock markets. The pair CAC-DAX is the one where extremal dependence peaks the most, thus suggesting a high level of synchronization and comovement of extreme losses in those markets over recent years.

Similar conclusions can be drawn from Figure 8, where we plot the conditional extremal coefficient, as defined in Section 2.3. The extremal coefficient is equal to $2-\chi$, and as such is equal to 2 under asymptotic independence, and takes values in $[1,2)$ under asymptotic dependence. Figure 8 permits comparison with the results of Poon, Rockinger and Tawn (2004), who calculated $\chi$ over subperiods. The red lines in Figure 8 represent the values from the analysis of Poon et al. for the subperiod November 1990-November 2001 [cf. Poon, Rockinger and Tawn (2004), Table 3]. Specifically, Poon et al. report the following values of $\chi$ for: CAC-DAX, 0.517 (0.037); FTSE-CAC, 0.532 (0.035) and FTSE-DAX, 0.459 (0.039), with standard errors in parentheses. As can be seen from Figure 8, the magnitudes of 

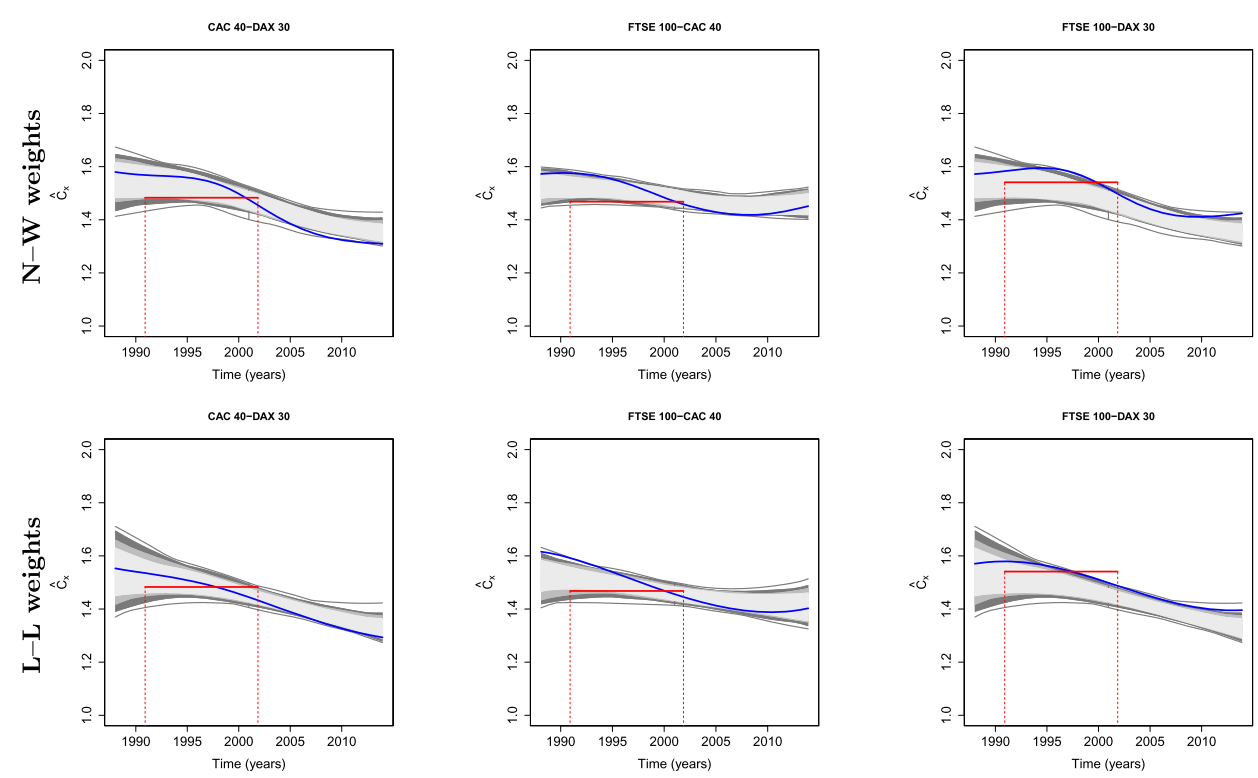

FIG. 8. Conditional extremal coefficients (solid blue lines) and functional boxplots (gray shadows) showing the 50\%, 75\%, and 95\% central regions [as defined by Sun and Genton (2011)] based on 1000 bootstrap samples. The red lines represent the values from the analysis of Poon et al. for the subperiod November 1990-November 2001 [cf. Poon, Rockinger and Tawn (2004), Table 3].

the extremal coefficients estimated by Poon et al. are in reasonable agreement with the ones computed with our methods when uncertainty is taken into account.

6. Final comments. This paper develops methods for modeling nonstationary extremal dependence structures, motivated by the need to assess the comovement of extreme losses in some leading European stock markets over recent years. Although there are many studies analyzing stock market integration over time [see, e.g., Brooks and Del Negro (2004, 2005), Forbes and Rigobon (2002), Karolyi and Stulz (1996), King, Sentana and Sushil (1994), Longin and Solnik (1995, 2001), Rua and Nunes (2009)], few attempts have been made to assess the dynamics of extreme value dependence of stock market returns over time. An exception in this regard is the paper of Poon, Rockinger and Tawn (2003), which provides evidence suggesting increasing levels of extremal dependence for CAC, DAX, and FTSE, although their analysis is essentially exploratory. The analysis performed in this paper reveals a more complete picture of this temporally-changing dependence.

Two related approaches to the current work are the so-called spectral density ratio model of de Carvalho and Davison (2014) and the spectral density regression model of Castro-Camilo and de Carvalho (2017). While flexible, these approaches only apply to the setting where there are several pseudo-angles corresponding to the same value of the predictor-and thus they are inappropriate for our applied 
setting of interest. Our methods are more resilient in the sense that they do not require a sample of pseudo-angles for each value of the covariate, but apply more generally to a regression setting where each covariate value may only have a single corresponding pseudo-angle. Recent preprints of Escobar-Bach, Goegebeur and Guillou (2016) and Mhalla, Chavez-Demoulin and Naveau (2017) suggest methods for estimating Pickands dependence function under covariate dependence, offering alternative approaches to those presented herein.

Computational experiments suggest that U-shaped angular surfaces are much more difficult to fit. Whilst absolute errors may become large at the boundaries when we have an unbounded density, when this is translated to other quantities ( $H_{x}$ or $A_{x}$ ), the errors will be much less noticeable. Our methods have been developed with the setting of asymptotic dependence in mind, but certainly there is room for developing methodology for conditional modeling under asymptotic independence. Indeed, in common with any approach based on multivariate extreme value distributions, a limitation with our methods is that they will overestimate risk if data are asymptotically independent. Figure 5 gave some indication of possible asymptotic independence near the beginning of the analysis period. As such, the need for developing conditional models able to cope with both asymptotic dependence and asymptotic independence is of utmost importance.

Acknowledgements. We thank the Editor, Associate Editor, and two anonymous referees. We extend our thanks to António Rua, Vanda Inácio de Carvalho, and Claudia Wehrhahn for helpful discussions. Part of this work was written while D. Castro-Camilo was visiting the University of Cambridge-Statistical Laboratory, and while M. de Carvalho was visiting Banco de Portugal.

\section{SUPPLEMENTARY MATERIAL}

\section{Supplementary Monte Carlo evidence and empirical reports.}

(DOI: 10.1214/17-AOAS1089SUPP; .pdf). The supplement includes additional simulation results, descriptive statistics for daily stock index negative returns, and further empirical analysis using the NGARCH-filtered residuals and LSCV bandwidths.

\section{REFERENCES}

ACAR, E. F., CRAIU, R. V. and YAO, F. (2011). Dependence calibration in conditional copulas: A nonparametric approach. Biometrics 67 445-453. MR2829013

Beirlant, J., Goegebeur, Y., Teugels, J. and Segers, J. (2004). Statistics of Extremes: Theory and Applications. Wiley, Chichester. MR2108013

Brooks, R. and DEL NeGRO, M. (2004). The rise in comovement across national stock markets: Market integration or IT bubble? J. Empir. Finance 11 659-680.

Brooks, R. and Del Negro, M. (2005). Country versus region effects in international stock returns. J. Portf. Manag. 31 67-72. 
BÜtTNER, D. and Hayo, B. (2011). Determinants of European stock market integration. Econ. Syst. 35 574-585.

Castro-Camilo, D. and de Carvalho, M. (2017). Spectral density regression for bivariate extremes. Stoch. Environ. Res. Risk Assess. 31 1603-1613.

Castro-Camilo, D., de Carvalho, M. and Wadsworth, J. (2018). Supplement to "Timevarying extreme value dependence with application to leading European stock markets." DOI:10.1214/17-AOAS1089SUPP.

Chavez-Demoulin, V. and Davison, A. C. (2005). Generalized additive modelling of sample extremes. J. Roy. Statist. Soc. Ser. C 54 207-222. MR2134607

Chen, S. X. (1999). Beta kernel estimators for density functions. Comput. Statist. Data Anal. 31 131-145. MR1718494

Cline, D. B. H. (1988). Admissible kernel estimators of a multivariate density. Ann. Statist. 16 1421-1427. MR0964931

Coles, S. (2001). An Introduction to Statistical Modeling of Extreme Values. Springer, London. MR1932132

Coles, S. G. and TAWn, J. A. (1991). Modelling extreme multivariate events. J. Roy. Statist. Soc. Ser. B 53 377-392. MR1108334

DasGupta, A. (2008). Asymptotic Theory of Statistics and Probability. Springer, New York. MR2664452

Davison, A. C. and Smith, R. L. (1990). Models for exceedances over high thresholds. J. Roy. Statist. Soc. Ser. B 52 393-442. MR1086795

DE CARvalho, M. (2017). Statistics of extremes: Challenges and opportunities. In Extreme Events in Finance. Wiley Handb. Finance Eng. Econom. 195-213. Wiley, Hoboken, NJ. MR3617263

De Carvalho, M. and Davison, A. C. (2014). Spectral density ratio models for multivariate extremes. J. Amer. Statist. Assoc. 109 764-776. MR3223748

de Carvalho, M., Oumow, B., Segers, J. and Warchoł, M. (2013). A Euclidean likelihood estimator for bivariate tail dependence. Comm. Statist. Theory Methods 42 1176-1192. MR3031275

DE HAAN, L. and RESNICK, S. I. (1977). Limit theory for multivariate sample extremes. Z. Wahrsch. Verw. Gebiete 40 317-377.

EASTOE, E. F. (2009). A hierarchical model for non-stationary multivariate extremes: A case study of surface-level ozone and $\mathrm{NO}_{\mathrm{x}}$ data in the UK. Environmetrics 20 428-444. MR2834808

Eastoe, E. F. and TAWN, J. A. (2009). Modelling non-stationary extremes with application to surface level ozone. J. R. Stat. Soc. Ser. C 58 25-45. MR2662232

ENGLE, R. F. (1982). Autoregressive conditional heteroscedasticity with estimates of the variance of United Kingdom inflation. Econometrica 50 987-1007. MR0666121

Escobar-Bach, M., Goegebeur, J. and Guillou, A. (2016). Local robust estimation of the Pickands dependence function. Submitted.

Fermanian, J.-D. and Wegkamp, M. H. (2012). Time-dependent copulas. J. Multivariate Anal. 110 19-29. MR2927507

FORBES, K. and RigOBON, R. (2002). No contagion, only interdependence: Measuring stock market comovements. J. Finance $572223-2261$.

FRATZSCHER, M. (2002). Financial market integration in Europe: On the effects of EMU on stock markets. Int. J. Fin. Econ. 7 165-193.

GeEnEns, G. (2014). Probit transformation for kernel density estimation on the unit interval. J. Amer. Statist. Assoc. 109 346-358. MR3180568

Gudendorf, G. and Segers, J. (2010). Extreme-value copulas. In Copula Theory and Its Applications. Lect. Notes Stat. Proc. 198 127-145. Springer, Heidelberg. MR3051266

HÄRdLE, W. (1990). Applied Nonparametric Regression. Cambridge Univ. Press, Cambridge. MR1161622 
Hardouvelis, G. A., Malliaropulos, D. and Priestley, R. (2006). EMU and European stock market integration. J. Bus. 79 365-392.

Hastie, T., Tibshirani, R. and Friedman, J. (2001). The Elements of Statistical Learning: Data Mining, Inference, and Prediction. Springer, New York. MR1851606

HefFERnAN, J. E. (2000). A directory of coefficients of tail dependence. Extremes 3 279-290. MR1856201

HEFFERNAN, J. E. and TAWN, J. A. (2004). A conditional approach for multivariate extreme values. J. R. Stat. Soc. Ser. B 66 497-546. MR2088289

Huser, R. and GENTON, M. G. (2016). Non-stationary dependence structures for spatial extremes. J. Agric. Biol. Environ. Stat. 21 470-491. MR3542082

JAMES, H. (2012). Making the European Monetary Union. Harvard Univ. Press, Cambridge MA.

Jonathan, P., EwAns, K. and RANDEll, D. (2014). Non-stationary conditional extremes of northern North Sea storm characteristics. Environmetrics 25 172-188. MR3200308

Jones, M. C. and HENDERSON, D. A. (2007). Kernel-type density estimation on the unit interval. Biometrika 94 977-984. MR2416803

KAROlYi, G. A. and StUlz, R. M. (1996). Why do markets move together? An investigation of US-Japan stock return comovements. J. Finance 51 951-986.

Kim, S. J., Moshirian, F. and WU, E. (2005). Dynamic stock market integration driven by the European monetary union: An empirical analysis. J. Bank. Fin. 29 2475-2502.

KIM, S., SHEPHARD, N. and CHIB, S. (1998). Stochastic volatility: Likelihood inference and comparison with ARCH models. Rev. Econ. Stud. 65 361-393.

King, M., Sentana, E. and Sushil, W. (1994). Volatility and links between national stock markets. Econometrica 62 901-933.

Koenker, R. (2005). Quantile Regression. Cambridge Univ. Press, Cambridge. MR2268657

LANe, P. R. (2012). The European sovereign debt crisis. J. Econ. Persp. $2649-67$.

LONGIN, F. and SOLNIK, B. (1995). Is the correlation in international equity returns constant: 19601990? J. Int. Money Financ. 14 3-26.

LONGIN, F. and SOLNIK, B. (2001). Extreme correlation of international equity market. J. Finance 56 649-676.

Mhalla, L., Chavez-Demoulin, V. and Naveau, P. (2017). Non-linear models for extremal dependence. J. Multivariate Anal. 159 49-66. MR3668547

Nadaraya, E. A. (1964). On estimating regression. Theory Probab. Appl. 9 141-142.

Natural Environment Research Council (1975). Flood Studies Report. Natural Environment Research Council, London.

PATTON, A. J. (2006). Modelling asymmetric exchange rate dependence. Internat. Econom. Rev. 47 527-556. MR2216591

PICKANDS, J. III (1981). Multivariate extreme value distributions. In Proceedings of the 43rd Session of the International Statistical Institute, Vol. 2 (Buenos Aires, 1981) 49 859-878, 894-902. MR0820979

POLANSKY, A. M. (2001). Bandwidth selection for the smoothed bootstrap percentile method. Comput. Statist. Data Anal. 36 333-349. MR1836205

Poon, S.-H., Rockinger, M. and TAWn, J. A. (2003). Modelling extreme-value dependence in international stock markets. Statist. Sinica 13 929-953.

Poon, S.-H., Rockinger, M. and TAWn, J. (2004). Extreme-value dependence in financial markets: Diagnostics, models and financial implications. Rev. Financ. Stud. 17 581-610.

Resnick, S. I. (2007). Heavy-Tail Phenomena: Probabilistic and Statistical Modeling. Springer, New York. MR2271424

RUA, A. and NUNES, L. C. (2009). International comovement of stock market returns: A wavelet analysis. J. Empir. Finance 16 632-639.

Silverman, B. W. and Young, G. A. (1987). The bootstrap: To smooth or not to smooth? Biometrika 74 469-479. MR0909352 
Sun, Y. and Genton, M. G. (2011). Functional boxplots. J. Comput. Graph. Statist. 20 316-334. MR2847798

TAWN, J. A. (1988). Bivariate extreme value theory: Models and estimation. Biometrika 75 397-415. MR0967580

TAWN, J. A. (1990). Modelling multivariate extreme value distributions. Biometrika 77 245-253.

VERAVERBEKE, N., OMELKA, M. and GiJbels, I. (2011). Estimation of a conditional copula and association measures. Scand. J. Stat. 38 766-780. MR2859749

WAND, M. P. and Jones, M. C. (1995). Kernel Smoothing. Chapman \& Hall, London. MR1319818

WATSON, G. S. (1964). Smooth regression analysis. Sankhyā Ser. A 26 359-372. MR0185765

D. CASTRO-CAMilo

M. DE CARVALHO

CEMSE DIVISION

SCHOOL OF MATHEMATICS

KING ABDULlaH UNIVERSITY

UNIVERSITY OF EDINBURGH

OF SCIENCE AND TECHNOLOGY

EDINBURGH

THUWAL

Kingdom of SAUdi ARABIA

UNITED KINGDOM

E-MAIL: daniela.castro@kaust.edu.sa

E-MAIL: miguel.decarvalho@ed.ac.uk

\author{
J. WADSWORTH \\ DEPARTMENT OF MATHEMATICS AND STATISTICS \\ LANCASTER UNIVERSITY \\ LANCASTER \\ UNITED KINGDOM \\ E-MAIL: j.wadsworth@lancaster.ac.uk
}

